\title{
Decoherence of multi-dimensional entangled coherent states
}

\author{
S. J. van Enk \\ Bell Labs, Lucent Technologies \\ 600-700 Mountain Ave, Murray Hill, NJ 07974
}

(Dated: August 1, 2018)

\begin{abstract}
For entangled states of light both the amount of entanglement and the sensitivity to noise generally increase with the number of photons in the state. The entanglement-sensitivity tradeoff is investigated for a particular set of states, multi-dimensional entangled coherent states. Those states possess an arbitrarily large amount of entanglement $E$ provided the number of photons is at least of order $2^{2 E}$. We calculate how fast that entanglement decays due to photon absorption losses and how much entanglement is left. We find that for very small losses the amount of entanglement lost is equal to $2 / \log (2) \approx 2.89$ ebits per absorbed photon, irrespective of the amount of pure-state entanglement $E$ one started with. In contrast, for larger losses it tends to be the remaining amount of entanglement that is independent of $E$. This may provide a useful strategy for creating states with a fixed amount of entanglement.
\end{abstract}

\section{INTRODUCTION}

Entanglement lies at the heart of many quantum information processing protocols [1]. In order to generate entangled states one typically envisages a process that in the ideal case would lead to the desired state with the desired amount of entanglement. Imperfections such as noise and losses, however, degrade the quality of the state and diminish the amount of entanglement created. As a consequence, the task for which the entangled state is created (say, teleportation [2]) will be accomplished only partially. An alternative strategy for generating a certain amount of entanglement would be the following: use a process that in the ideal case would generate (much) more entanglement than needed, but that produces the desired amount in the realistic imperfect case.

If it were increasingly difficult to generate larger amounts of entanglement, that strategy would not be very useful. Fortunately, it turns out that for light beams a standard optical nonlinearity [20] allows one to generate more entanglement when the interaction time (or length) $\tau$ is shorter: one generates $\log _{2} M$ ebits in a time $\tau=1 / M[5]$. This effect exploits some of the peculiar properties of entanglement in infinite-dimensional Hilbert spaces [6]. We may use that effect in the following way: take a very short time (or length) $\tau=1 / M$ to generate a bipartite $M \times M$ dimensional pure state of two modes with $\log _{2} M$ ebits of entanglement, with $M$ large. Since the interaction time is assumed short we may neglect decoherence during that part of the procedure and consequently the state at that point is still pure. By the time we will actually use the state for some quantuminformation processing protocol, there will have been decoherence, turning the pure state into a mixed state $\rho$. The most relevant type of decoherence for light beams is that due to photon absorption losses, and that is the only type of decoherence we will consider here. We are interested in the amount of bipartite entanglement between the two modes [7] in the state $\rho$.

Since $\rho$ will typically still be a very high-dimensional state, we use a measure of entanglement that can actually be computed efficiently even in that case: the logarithmic negativity $E_{N}(\rho)[8]$. That quantity can be linked to two operational measures of entanglement, demonstrating the possible use of the mixed-state entanglement in $\rho$. On the one hand $E_{N}(\rho)$ determines a bound (through the negativity in fact) on the fidelity that can be achieved in teleportation using a single copy of $\rho$, on the other it gives a bound on the amount of distillable (pure-state) [9] entanglement present in many copies of $\rho$ (see Ref. 8] for details).

An alternative method to create certain desired entangled states of light was discussed in 10]: that method is very similar in spirit to that analyzed here but still relies on producing a pure state. The first part of the procedure is the same as above and consists of generating an $M \times M$-dimensional pure entangled state (with $M$ large) of two modes. But subsequently one performs appropriate local measurements on both modes to (approximately and probabilistically) project the state down to a $2 \times 2$-dimensional state of the form

$$
|\alpha\rangle \otimes|\alpha\rangle+\exp (i \phi)|-\alpha\rangle \otimes|-\alpha\rangle,
$$

where $|\alpha\rangle$ is a coherent state, and where the amplitude $\alpha$ is not too small, such that $|\alpha\rangle$ and $|-\alpha\rangle$ are to a very good approximation orthogonal. In that case, the states (1) possess one ebit of entanglement for any value of the phase $\phi$ and such states can then be used for teleportation 11], quantum computing [12], quantum communication [13], and testing nonlocality [14].

Generally speaking, the sensitivity to noise increases with the number of photons in the state. This was noted, in particular, in experiments on generating Schrödinger cat states of the form $|\alpha\rangle+\exp (i \phi)|-\alpha\rangle[15,16 \mid$. It is also true for the related entangled coherent states [11, 17], and this issue was subsequently investigated in more detail and generality for light beams in 18]. For an even more general discussion of decoherence of macroscopic and microscopic objects, see for example [19]. Thus, while increasing $M$ leads to more entanglement it also leads to the state being more sensitive to decoherence, as the number of photons needed to create that much entan- 
glement increases as $M^{2}$. That is, one expects the state to lose entanglement at a rate that increases with $M$. Thus, one may expect an optimum number of photons for fixed interaction time and fixed amount of decoherence to exist that maximizes the amount of entanglement left after decoherence. Confirming that expectation is another aim of this paper.

\section{ANALYTICAL RESULTS}

\section{A. Multi-dimensional entangled coherent states}

We first review some of the results of [5] that we need here. Suppose one starts out with a coherent state $|\sqrt{2} \alpha\rangle$ and one lets the state propagate through a Kerr nonlinear medium, described by a Hamiltonian of the form $H=a^{\dagger 2} a^{2}$ for a time $\tau$ equal to $1 / M$, with $M$ an integer. Subsequently one splits the resulting state on a $50 / 50$ beamsplitter with the vacuum. The state of the two output modes so generated is of the form

$$
|\Phi\rangle=\sum_{q=1}^{M} f_{q}\left|\Phi_{q}\right\rangle \otimes\left|\Phi_{q}\right\rangle,
$$

where the states $\left|\Phi_{q}\right\rangle$ are coherent states with amplitude $\alpha$ and phases $-2 \pi q / M$,

$$
\left|\Phi_{q}\right\rangle=|\alpha \exp (-2 \pi i q / M)\rangle
$$

The states (2) generalize the states (11) and may be called multi-dimensional entangled coherent states. For ease of notation, here and in the following we suppress the dependence of the states $\left|\Phi_{q}\right\rangle$ and similar states defined below on the parameters $\alpha$ and $M$. Up to irrelevant overall phase factors the coefficients $f_{q}$ are given by

$$
\begin{aligned}
& f_{q}=\frac{1}{\sqrt{M}} \exp \left(\frac{\pi i q(q+1)}{M}\right), M \text { odd } \\
& f_{q}=\frac{1}{\sqrt{M}} \exp \left(\frac{\pi i q^{2}}{M}\right), M \text { even }
\end{aligned}
$$

The states (2) potentially possess $\log _{2} M$ ebits of entanglement, namely when $\alpha$ is sufficiently large to make the states $|\alpha \exp (-2 \pi i q / M)\rangle$ and $\left|\alpha \exp \left(-2 \pi i q^{\prime} / M\right)\right\rangle$ nearly orthogonal for $q \neq q^{\prime}$. This requires that $|\alpha|^{2}$ be at least of order $M^{2}$. More precisely, if we choose a small number $\delta \ll 1$ the requirement

$$
\left|\left\langle\Phi_{q} \mid \Phi_{q+1}\right\rangle\right|^{2}=\delta
$$

gives

$$
|\alpha|^{2} \approx \frac{\log (1 / \delta)}{4 \pi^{2}} M^{2},
$$

for large $M$. This follows from the expression for the overlap of two coherent states with complex amplitudes $\alpha$ and $\beta$

$$
\langle\alpha \mid \beta\rangle=\exp \left(\alpha^{*} \beta-|\alpha|^{2} / 2-|\beta|^{2} / 2\right)
$$

Thus, the shorter the interaction time $\tau=1 / M$, the more entanglement the states (2) contain, provided the number of photons is sufficiently large.

\section{B. Decoherence due to photon absorption}

The decoherence of a mode due to photon absorption losses can be described by a parameter $\eta$ that gives the fraction of photons surviving the absorption process. The loss process is determined by its effect on coherent states by

$$
|\alpha\rangle \otimes|0\rangle_{E} \rightarrow|\sqrt{\eta} \alpha\rangle \otimes|\sqrt{1-\eta} \alpha\rangle_{E},
$$

where $E$ refers to the environment, which will be traced out. We have a bipartite state on two modes, and when we assume both modes to decohere in the same way, the pure state $|\Phi\rangle$ is turned into a mixture of the form

$$
\begin{array}{r}
\rho=\sum_{q=1}^{M} \sum_{p=1}^{M} f_{q} f_{p}^{*}\left(\left\langle\Psi_{p} \mid \Psi_{q}\right\rangle\right)^{2} \\
\left|\tilde{\Phi}_{q}\right\rangle\left\langle\tilde{\Phi}_{p}|\otimes| \tilde{\Phi}_{q}\right\rangle\left\langle\tilde{\Phi}_{p}\right| .
\end{array}
$$

Here we defined two new states, related to those defined in (3). First, due to decoherence the amplitude $\alpha$ is reduced by a factor of $\eta$, and hence we used the states

$$
\left|\tilde{\Phi}_{q}\right\rangle=|\sqrt{\eta} \alpha \exp (-2 \pi i q / M)\rangle
$$

to expand $\rho$ in. Second, the state of the environment can be expanded, likewise, in terms of the states

$$
\left|\Psi_{q}\right\rangle=|\sqrt{1-\eta} \alpha \exp (-2 \pi i q / M)\rangle .
$$

After tracing out the environment the overlaps of such states appear in the expression for $\rho$, hence the appearance of the factor $\left(\left\langle\Psi_{p} \mid \Psi_{q}\right\rangle\right)^{2}$ in (10).

\section{Decoherence of entanglement}

In order to determine the entanglement in the state $\rho$ as a function of $\eta, \alpha, M$, we use, as announced, the logarithmic negativity as measure of entanglement [8]. We first have to express the density matrix in some orthogonal basis. This can be accomplished as follows: first define the $M$-by- $M$ Gram matrix $G$ by its entries

$$
G_{p q}=\left\langle\tilde{\Phi}_{p} \mid \tilde{\Phi}_{q}\right\rangle
$$

Then suppose we have chosen $M$ orthogonal states $\left|x_{i}\right\rangle$ for $i=1 \ldots M$ in terms of which we expand

$$
\left|\tilde{\Phi}_{q}\right\rangle=\sum_{i=1}^{M} A_{q i}\left|x_{i}\right\rangle .
$$

Similarly, we expand the density matrix $\rho$ in terms of the basis formed by the set

$$
\left|x_{i}\right\rangle\left\langle x_{j}|\otimes| x_{k}\right\rangle\left\langle x_{l}\right| .
$$


The matrix $A$ can be expressed in terms of the Gram matrix by using the relation

$$
G_{p q}=\sum_{j=1}^{M} A_{q j} A_{j p}
$$

Namely, in brief notation, we have

$$
A=\sqrt{G^{T}}
$$

with the superscript $T$ denoting the transpose. Here we used the fact that $G$ is a Hermitian matrix with positive eigenvalues, so that $A$, too, can be chosen as a Hermitian matrix with positive eigenvalues. Defining a matrix $C$ by its elements

$$
C_{m n, q}=f_{q} A_{q m} A_{q n}
$$

we can write the density matrix $\rho$ as

$$
\rho=C G_{2} C^{T}
$$

where $G_{2}$ is the matrix defined by

$$
\left(G_{2}\right)_{p q}=\left(\left\langle\Psi_{p} \mid \Psi_{q}\right\rangle\right)^{2}
$$

The partial transpose of $\rho$ with respect to the first mode and with respect to the basis (15) is denoted by $\rho^{T_{1}}$ and is defined through

$$
\rho_{k l, m n}^{T_{1}}=\rho_{m l, k n} .
$$

The logarithmic negativity of $\rho$, finally, is given by

$$
E_{N}(\rho)=\log _{2}\left\|\rho^{T_{1}}\right\|
$$

with $\|$.$\| denoting the trace norm, which for Hermitian$ operators is equal to the sum of the absolute values of the eigenvalues. The result (22) is exact and can be calculated numerically in a straightforward way by using standard linear algebra. On the other hand, the size of the matrix $\rho$ may become too large for large $M$ to be useful in numerical computations. Here we use Matlab, and whenever $M \leq 50$ we use the exact result (22). In other cases we can use certain approximations valid under the appropriate conditions.

\section{Some approximations}

In the case that the states $\left|\tilde{\Phi}_{q}\right\rangle$ are almost orthogonal, we can approximate $E_{N}$ as follows. First, the partial transpose of $\rho$ is approximated by

$$
\begin{array}{r}
\rho^{T_{1}} \approx \sum_{q=1}^{M} \sum_{p=1}^{M} f_{q} f_{p}^{*}\left(\left\langle\Psi_{p} \mid \Psi_{q}\right\rangle\right)^{2} \\
\left|\tilde{\Phi}_{p}\right\rangle\left\langle\tilde{\Phi}_{q}|\otimes| \tilde{\Phi}_{q}\right\rangle\left\langle\tilde{\Phi}_{p}\right| .
\end{array}
$$

Then the $M^{2}$ eigenvalues of this matrix can be written as

$$
\begin{aligned}
\lambda_{k, m}^{ \pm}= & \pm \frac{1}{M} \exp \left(-(1-\eta)|\alpha|^{2}|\exp (2 \pi i(k-m) / M)-1|^{2}\right) \\
& \text { for }(1 \leq k<m \leq M) \\
\lambda_{k}= & \frac{1}{M} \text { for }(1 \leq k \leq M)
\end{aligned}
$$

Hence

$$
E_{N}(\rho) \approx \log _{2}[1+F]
$$

where

$$
F=\sum_{k=1}^{M-1} \exp \left(-(1-\eta)|\alpha|^{2}|\exp (2 \pi i k / M)-1|^{2}\right)
$$

We can further approximate this when

$$
\epsilon=1-\eta
$$

is a small number, $\epsilon \ll 1$. In fact, when not only $\epsilon$ is small but $\epsilon|\alpha|^{2}$ is small as well, we can simply expand the exponentials appearing in the expression for $F$ to obtain

$$
\begin{aligned}
E_{N}(\rho) & \approx \log _{2}\left[M-2 \epsilon|\alpha|^{2}(M-1)\right] \\
& \approx \log _{2} M-\epsilon|\alpha|^{2} \frac{2}{\log (2)} \frac{M-1}{M}
\end{aligned}
$$

When $\epsilon$ becomes larger, such that $\epsilon|\alpha|^{2}$ is no longer small, we can make a different approximation in the case that relation (7) holds with $\delta \ll 1$ some fixed small number. In this case, $F$ can be rewritten as

$$
F=\sum_{k=1}^{M-1} \exp \left(-\epsilon \log (1 / \delta) M^{2}|\exp (2 \pi i k / M)-1|^{2} /(2 \pi)^{2}\right)
$$

This sum cannot be performed analytically, but we can approximate it as

$$
F \approx \sum_{k=1}^{M-1} \delta^{k^{2} \epsilon}
$$

which is valid for $\epsilon$ not too small. Since $\delta \ll 1$ only terms with sufficiently small values of $k$ contribute. In particular, if we simply cut off the summation when $\delta^{k^{2} \epsilon}$ becomes smaller than some number $D$, i.e., at $k=$ $\sqrt{\log (D) / \log (\delta)} \sqrt{1 / \epsilon}$, then the entanglement $E_{N}(\rho)$ is of the form

$$
\left.E_{N}(\rho) \approx \log _{2}\left[1+\sqrt{D^{\prime} /(\epsilon \log (\delta)}\right)\right]
$$

We checked this approximation numerically and $D^{\prime}$ was determined to be around $D^{\prime} \approx \pi$. 


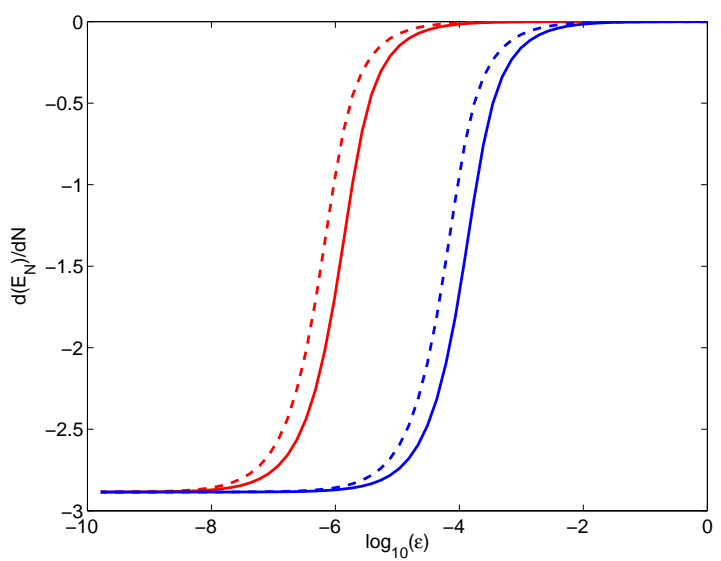

FIG. 1: Loss of entanglement per photon $d E_{N} / d N$ as a function of $\log _{10} \epsilon$. Two curves on the right correspond to $M=200$, the two on the left to $M=2000$. The solid curves correspond to $\delta=10^{-2}$ and the dashed curves to $\delta=10^{-4}$. This plot uses the approximation (25).

\section{NUMERICAL RESULTS}

Here we use the exact result (22) and the approximate results (25), (28), and (31) to investigate the behavior of entanglement as functions of various different parameters.

When $\alpha$ is sufficiently large and $\eta=1$ one will have $\log _{2} M$ ebits of entanglement. But how quickly does one lose that possibly large amount of entanglement when there is a small amount of decoherence? The average number of photons absorbed from each mode is

$$
\Delta N=\epsilon|\alpha|^{2} .
$$

The change in entanglement from pure to mixed state,

$$
\Delta E_{N}=E_{N}(\rho)-E_{N}(|\Phi\rangle\langle\Phi|)
$$

is, for very small losses, proportional to $\Delta N$ and is given by $2 / \log (2) \approx 2.89$ ebits per photon:

$$
\Delta E_{N} \approx-\frac{2}{\log (2)} \frac{M-1}{M} \Delta N,
$$

where we used (28). This shows how fragile the initial pure entangled states are when they contain a large number of photons. Even when a tiny fraction $\epsilon$ of photons is lost, the number of photons lost may still be appreciable if $|\alpha|^{2}$ is large, and this may lead to a large degradation of entanglement.

When $\epsilon$ increases the entanglement loss per photon decreases. Using (25) [since we assume $\epsilon|\alpha|^{2}$ is not small we cannot use (28); we do assume $\delta \ll 1$ now] we plot $d E_{N} / d N$ as a function of $\log _{10} \epsilon$ in Fig. 1. The 4 curves plotted in Fig. 1 are shifted versions of one another: in fact, they are really only functions of $\Delta N=\epsilon|\alpha|^{2}$.

The approximation (31), too, depends only on $\Delta N$, not on $\delta$ or $\epsilon$ separately. In addition the change in entanglement $\Delta E_{N}$ does not depend on $M$ either since both

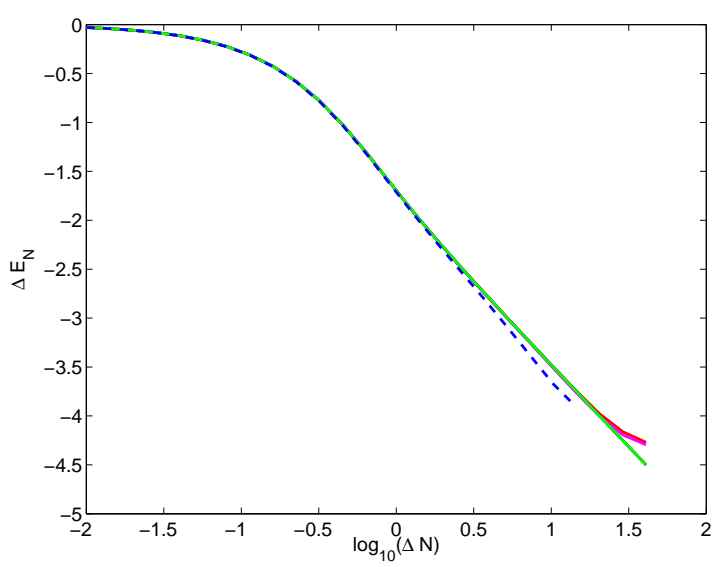

FIG. 2: Change in logarithmic negativity, $\Delta E_{N}$ as a function of the change in number of photons, $\Delta N$. There are in fact 5 curves here, several of which overlap to make them indistinguishable. The dashed curve is for $M=20$ and $\delta=0.2$. The solid, almost overlapping curves, are for $\delta=0.01$ and $M=20$, and for $\delta=10^{-4}$ with $M=20,200$, and 2000.

$E_{N}(\rho)$ and $E_{N}(|\Phi\rangle\langle\Phi|)$ depend on $M$ in the same way. To verify this behavior and to see whether this behavior extends to parameter regimes not covered by the conditions $\delta \ll 1$ and $\epsilon \ll 1$ we plot in Fig. 2 the change in entanglement $\Delta E_{N}$ as a function of $\Delta N$ for various different parameters. For large $M$ we use the approximation (26), but for $M=20$ we use the exact result (22). The plot confirms the predicted behavior: for $\delta$ not too large the change in entanglement is only a function of $\Delta N$, but not of $M$, or $\epsilon$ or $\delta$ independently. For $\delta=0.2$ (and $M=20$ ) there is only a small deviation from that universal behavior.

Fig. 3 plots, in contrast, not the change in entanglement but the entanglement $E_{N}(\rho)$ left after decoherence. The parameter regime considered is very different as $\epsilon$ is no longer assumed very small. We do assume $\delta \ll 1$ so that we can use the approximation (25). From the approximation (31) to that approximation we see that $E_{N}(\rho)$ no longer depends on $M$, provided $M$ is not too small. That behavior, too, is confirmed in Fig. 3, where curves for $M=20$ (recall that whenever $M \leq 50$ the exact result (22) is used) are seen to overlap with one for $M=20,000$. For smaller values of $M$ there is a clear dependence on $M$, as is illustrated for $M=2,3,5$. From Fig. 3 one notices that at reasonable (i.e. realistic) values of $\epsilon$ the amount of entanglement left is less than one unit, even if we started out with a state with $\log _{2} M \approx 14.3$ for $M=20,000$. However, that result is not optimized for the number of photons $|\alpha|^{2}$ in the initial state. In particular, since we chose $\delta$ to be small, $|\alpha|^{2}$ is roughly equal to $M^{2}$.

In order to see whether smaller numbers of photons may leave more entanglement after decoherence (since one expects the decoherence to have a less strong influence when there are fewer photons even if the initial pure state does not quite have the full $\log _{2} M$ ebits of 


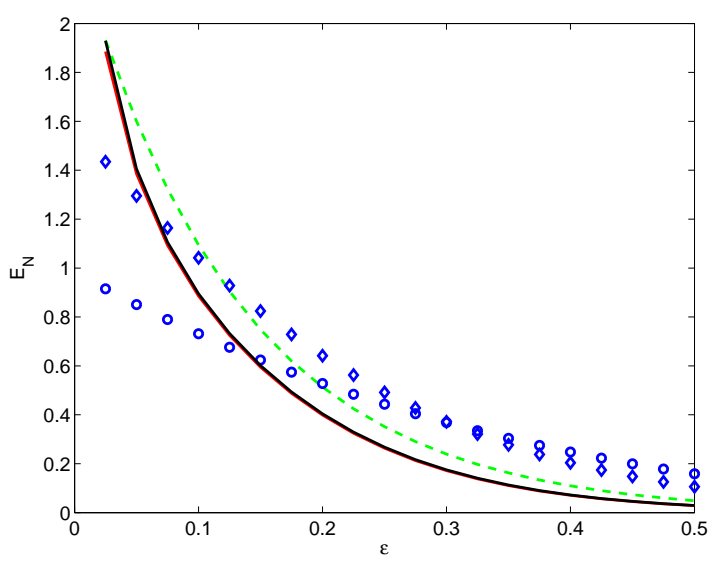

FIG. 3: Logarithmitic negativity $E_{N}(\rho)$ as a function of $\epsilon=1-\eta$. There are two solid curves almost completely overlapping that correspond to $M=20$ and $M=20000$, demonstrating that the amount of entanglement left after decoherence is insensitive to $M$ when $M$ is not too small. The dashed curve corresponds to $M=5$, the circles to $M=2$, the diamonds to $M=3$.

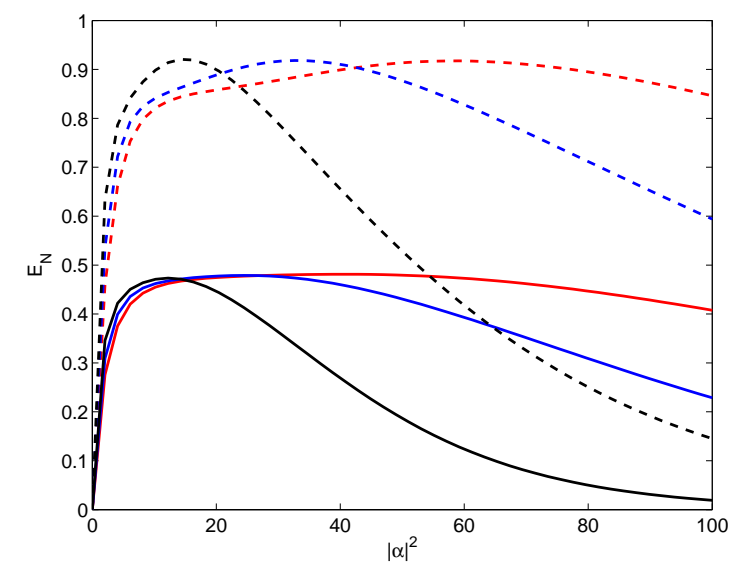

FIG. 4: Logarithmitic negativity $E_{N}(\rho)$ as a function of $|\alpha|^{2}$. There are three pairs of curves, each pair consisting of a solid curve, correpsonding to $\eta=0.7$, and a dashed curve (for $\eta=0.49$ ). The three pairs correspond to $M=20,30$, and 40, with the maxima of the curves with larger $M$ moving to larger $|\alpha|^{2}$.

entanglement), we plot in Fig. $4 E_{N}(\rho)$ as a function of $|\alpha|^{2}$ for various values of $M$. Here we cannot use the approximation (25) and hence use the exact calculation, which, as mentioned before, does restrict the calculation to $M \leq 50$. The plot shows that the optimum amount of entanglement remaining after decoherence is almost independent of the value of $M$, thus confirming the findings from Fig. 3. For example, for $\eta=0.7$ one is left with about 0.92 ebits of entanglement for the optimum values of $|\alpha|^{2}$ almost irrespective of how much entanglement the initial pure state had. On the other hand, there is a strong dependence on $|\alpha|^{2}$. Finally, the plot also shows that the decohered states become more robust against

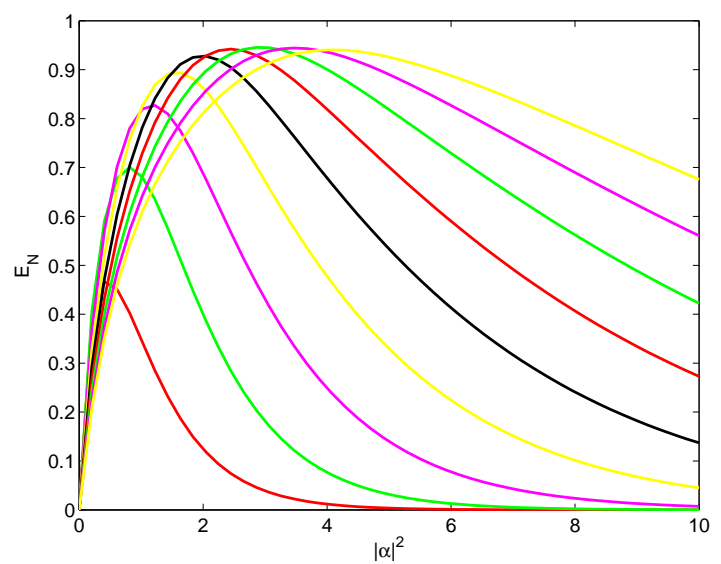

FIG. 5: Similar to Fig. 4: $E_{N}(\rho)$ as a function of $|\alpha|^{2}$ for $\eta=0.7$ and $M=2,3,4,5,6,7,8, \ldots 10$, where the maxima of the curves move to larger values of $|\alpha|^{2}$ for larger $M$.

photon absorption losses. For, even if one started out with a very large amount of (pure-state) entanglement, losing $30 \%$ of the photons $(\eta=0.7)$ leaves one with at best around 0.9 ebits of entanglement. But a further loss of $30 \%$ (so that $\eta=0.49$ ) of the photons merely decreases the entanglement by a factor of 2 .

In Fig. 5 we plot the same function but for smaller values of $M$. As it turns out, if one fixes $\eta=0.7$ the largest amount of entanglement one can be left with, starting out with multi-dimensional entangled coherent states, is in fact $E_{\max } \approx 0.95$ achieved by taking $M=8$ and $|\alpha|^{2} \approx 2.86$.

\section{CONCLUSIONS}

We considered the decoherence of multi-dimensional entangled coherent states due to photon absorption losses. Those states are bipartite entangled states of two modes, and are described by two independent parameters. One is $\alpha$, such that $|\alpha|^{2}$ gives the average number of photons in each mode. The other is $M$, an integer indicating the maximum possible amount of entanglement between the two modes, $\log _{2} M$. The amount of photon absorption is governed by an additional parameter, $\epsilon$, the fraction of photons absorbed from each mode. We calculated the amount of entanglement left after decoherence as a function of $\alpha, \epsilon$, and $M$. One useful quantity that can be constructed from the above parameters is $\Delta N=\epsilon|\alpha|^{2}$, the number of photons absorbed from each mode. Another useful quantity is $\delta$ defined as $\delta=\exp \left(-|\alpha|^{2}|\exp (2 \pi i / M)-1|^{2}\right)$, which determines the overlap between two coherent states of amplitude $\alpha$ and phase difference $2 \pi / M$. In terms of those quantities the conclusions are

1. For large $M$ the entanglement disappears at a rate given by $2 / \log (2) \approx 2.89$ ebits per photon as long as $\Delta N \ll 1$. 
2. For large $M$, small $\delta$ and not too large values of $\Delta N$ the change in entanglement is a function of $\Delta N$ alone.

3. For large $M$ and $\Delta N$, and for small $\delta$ it is the remaining amount of entanglement that is a function of $\Delta N$ alone.

4. In general there is an optimum number of photons $|\alpha|^{2}$ for fixed $\epsilon$ and $M$ that maximizes the amount of entanglement left after decoherence. For that optimum, $\delta$ is not very small (around 0.2 ).
As an example of point 4, we found that almost one ebit of entanglement can be obtained with $30 \%$ photon absorption by using a little less than 3 photons per mode for the initial pure state and $M=8$. The corresponding value of $\delta$ is about 0.19 . That is the maximum amount possible, but almost the same amount of entanglement (at least $95 \%$ of the optimum) can be obtained by using almost any value of $M$ and the appropriate value for $|\alpha|^{2} \approx M^{2} / 30$.
[1] M.A. Nielsen and I.L. Chuang, Quantum Computation and Quantum Information, Cambridge University Press (2000).

[2] C.H. Bennett et al., Phys. Rev. Lett., 70, 1895 (1993).

[3] B. Yurke and D. Stoler, Phys. Rev. Lett. 57, 13 (1986).

[4] B.C. Sanders, Phys. Rev. A 45, 6811 (1992).

[5] S.J. van Enk, Phys. Rev. Lett. 91, 017902 (2003).

[6] R. Clifton and H. Halvorson, Phys. Rev. A 61, 012108 (2000); J. Eisert, C. Simon, and M.B. Plenio, J. Phys. A 35, 3911 (2002); M. Keyl, D. Schlingemann, and R.F. Werner, Quant. Inf. Comput. 3, 281 (2003).

[7] P. Zanardi, Phys. Rev. A 65, 042101 (2002); S.J. van Enk, Phys. Rev. A 67, 022303 (2003); Y. Shi, Phys. Rev. A 67, 024301 (2003).

[8] G. Vidal and R.F. Werner, Phys. Rev. A 65, 032314 (2002).

[9] C.H. Bennett et al., Phys. Rev. Lett. 76, 722 (1996).

[10] H. Jeong et al., Phys. Rev. A 70, 061801(R) 2004.

[11] S.J. van Enk and O. Hirota, Phys. Rev. A 64, 022313 (2001).

[12] H. Jeong and M.S. Kim, Phys. Rev. A 65, 042305 (2002); T.C. Ralph, W.J. Munro, and G.J. Milburn, ibid. 68,
042319 (2003).

[13] S. Glancy, H.M. Vasconcelos, and T.C. Ralph, Phys. Rev. A 70, 022317 (2004).

[14] R. Filip, J. Řehaček and M. Dušek, quant-ph/0011006 D. Wilson, H. Jeong, and M.S. Kim, J. Mod. Optics 49, 851 (2002); H. Jeong et al. Phys. Rev. A 67, 012106 (2003).

[15] J.M. Raimond, M. Brune, and S. Haroche, Phys. Rev. Lett. 79, 1964 (1997).

[16] C.J. Myatt et al., Nature 403, 269 (2000).

[17] S-B Li and J-B Xu, Phys. Lett. A 309, 321 (2003).

[18] S.J. van Enk and O. Hirota, quant-ph/0412221

[19] D. Braun, F. Haake, and W.T. Strunz, Phys. Rev. Lett. 86, 2913 (2001); W.T. Strunz, F. Haake, and D. Braun, Phys. Rev. A 67, 022101 (2003); W.T. Strunz and F. Haake, ibid. 67, 022102 (2003).

[20] The Kerr nonlinearity arises, e.g., in nonlinear fibers: it has been known for a few decades that such a nonlinearity can be used to generate nonclassical states such as Schrödinger cat states [3] and entangled coherent states [4]. 